\title{
IDP-like Asteroids Formed Later than 5 Myr After Ca-Al-rich Inclusions
}

\author{
Marc Neveu ${ }^{1,2}$ (D) and Pierre Vernazza ${ }^{3}$ \\ ${ }^{1}$ University of Maryland, 4296 Stadium Dr., College Park, MD 20742, USA; marc.f.neveu@nasa.gov \\ ${ }^{2}$ NASA Goddard Space Flight Center, 8800 Greenbelt Rd., Greenbelt, MD 20770, USA \\ ${ }^{3}$ Aix-Marseille Université, CNRS, Laboratoire d'Astrophysique de Marseille, 38 Rue Frederic Joliot Curie, F-13013 Marseille, France \\ Received 2019 January 17; revised 2019 February 14; accepted 2019 March 5; published 2019 April 10
}

\begin{abstract}
The parent bodies of ordinary chondrites, carbonaceous CM chondrites, and interplanetary dust particles (IDPs) represent most of the mass of the solar system's small $(D \leqslant 250 \mathrm{~km})$ bodies. The times of formation of the ordinary and carbonaceous CM chondrite parent bodies have previously been pinpointed, respectively, to $\approx 2$ and 3-4 million years after calcium-aluminum-rich inclusions (CAIs). However, the timing of the formation of IDP parent bodies such as P- and D-type main-belt asteroids and Jupiter Trojans has not been tightly constrained. Here, we show that they formed later than 5-6 million years after CAIs. We use models of their thermal and structural evolution to show that their anhydrous surface composition would otherwise have been lost due to melting and icerock differentiation driven by heating from the short-lived radionuclide ${ }^{26} \mathrm{Al}$. This suggests that IDP-like volatilerich small bodies may have formed after the gas of the protoplanetary disk dissipated and thus later than the massive cores of the giant planets. It also confirms an intuitive increase in formation times with increased heliocentric distance, and suggests that there may have been a gap in time between the formation of carbonaceous chondrite (chondrule-rich) and IDP (chondrule-poor) parent bodies.
\end{abstract}

Key words: Kuiper belt: general - minor planets, asteroids: general - minor planets, asteroids: individual (Egeria, Eugenia, Hektor)

\section{Introduction}

Relating the compositional diversity among small bodies to their spatial distribution through time informs the understanding of solar system formation and evolution. Especially crucial to this understanding are S-, C-, and P- and D-type small bodies, which seem related, respectively, to ordinary chondrites (Burbine et al. 2002; Nakamura et al. 2011; Cloutis et al. 2014), carbonaceous chondrites (Johnson \& Fanale 1973; Lodders \& Osborne 1999; Lauretta et al. 2015; Perna et al. 2017), and interplanetary dust particles (IDPs; Sandford \& Bradley 1989; Liou et al. 1996; Bradley 2003; Vernazza et al. 2015), and represent the majority of the mass of observed small bodies (Pieters \& McFadden 1994; DeMeo \& Carry 2013), recovered fragments (Harvey \& Cassidy 1989; Burbine et al. 2002; Meteoritical Bulletin Database 2018), and estimated mass flux of extraterrestrial infall materials (Love \& Brownlee 1993).

The compositions of small bodies have deviated from those of the materials accreted due to processes such as differentiation, aqueous alteration, and/or thermal metamorphism. These require a heat source, understood since Urey (1955) to likely be the decay of short-lived radionuclides such as ${ }^{26} \mathrm{Al}$, which would have governed their early thermal evolution (e.g., Grimm \& McSween 1989; Bennett \& McSween 1996). Therefore, the present-day compositions observed among small bodies and their associated fragments result from (i) the accretion onset time, which controlled the amount of initial ${ }^{26} \mathrm{Al}$; (ii) the formation location, which controlled the initial rock/ice ratio; (iii) the size of the parent body; and (iv) the accretion duration, because accretion need not have been systematically instantaneous.

The accretion timing of major classes of small bodies can be constrained based on their (or their fragments') composition using telescopic observations, meteorite analyses, and thermal models. Inward of the snow line, the time of complete accretion has been constrained for the $\mathrm{H}$ chondrite parent body to $\sim 2 \pm 0.2$ Myr after Ca-Al-rich inclusions (CAIs; Henke et al. 2012; Monnereau et al. 2013; Blackburn et al. 2017) with a short accretion duration of $\leqslant 2 \times 10^{5} \mathrm{yr}$ (Monnereau et al. 2013). Spectroscopic investigation of $\mathrm{H}$-like asteroids confirmed those findings (Vernazza et al. 2014).

Observations of bodies bearing surface hydrated silicates (Usui et al. 2019) with a composition akin to $\mathrm{CM}$ or $\mathrm{CI}$ chondrites (e.g., Howard et al. 2009, 2011), suggest a later or longer accretion to avoid loss of volatiles from intense ${ }^{26} \mathrm{Al}$ heating, but not so late so water ice could have melted and altered silicates (Grimm \& McSween 1989; Bischoff 1998; Cohen \& Coker 2000; Young et al. 2003). To accrete the volatiles responsible for alteration, these bodies must have originated beyond the snow line (i.e., likely beyond Jupiter; Warren 2011; Budde et al. 2016; Kruijer et al. 2017; Desch et al. 2018). The coexistence of observed hydrated phases with dry silicates such as pyroxene and olivine (Howard et al. 2009 , 2011) suggests that aqueous alteration was limited either in time or by the availability of fluid (water-to-rock mass ratio below unity; Tomeoka \& Buseck 1985; Grimm \& McSween 1989; Zolensky et al. 1993; Rosenberg et al. 2001; Neveu et al. 2017).

Thermal evolution simulations suggest that melting and pervasive aqueous alteration can take place in small bodies forming 3-3.5 Myr after CAIs, but not for formation past $4 \mathrm{Myr}$ after CAIs (Bland \& Travis 2017). Accretion may have started earlier, perhaps as early as it did inward of the snow line, as suggested by evidence for material isotopically related to carbonaceous chondrites but having undergone thermal metamorphism or rock-metal differentiation. This includes clasts in CV chondrites (Weiss \& Elkins-Tanton 2013), iron meteorite groups IIC, IID, IIF, IIIF, and IVB (Kruijer et al. 2017), and Eagle Station pallasites (e.g., Greenwood et al. 2017). However, this material represents only a small fraction 
of the meteorite collection compared to the bulk of carbonaceous chondrites (Meteoritical Bulletin Database 2018), and does not undermine the conclusion that much of the material of carbonaceous chondrite parent bodies was likely accreted between 3 and $4 \mathrm{Myr}$ after CAIs.

Yet more distant, volatile-rich bodies such as IDP-like P- and D-type main-belt asteroids and Jupiter Trojans, as well as some closer-in C-types, show little to no evidence for surface hydration (Usui et al. 2019). Instead, their surfaces comprise likely indigenous anhydrous silicates (e.g., Emery et al. 2006; Vernazza et al. 2012, 2015, 2017) and water ice (Campins et al. 2010; Rivkin \& Emery 2010; Licandro et al. 2011; Hargrove et al. 2012; Takir \& Emery 2012). The higher bulk abundances of low-density volatiles (ices) in these bodies is also indicated by lower observed bulk densities, near $1500 \mathrm{~kg} \mathrm{~m}^{-3}$ but possibly as low as $800 \mathrm{~kg} \mathrm{~m}^{-3}$ (Vernazza \& Beck 2017; Pajuelo et al. 2018; Castillo-Rogez et al. 2019, and references therein). Those of the Jupiter Trojans Hektor and Patroclus are also in this range (Marchis et al. 2014; Emery et al. 2015 and references therein). The surfaces of more distant objects such as Centaurs and KBOs, the likely parent population of P/D-type asteroids and Jupiter Trojans (e.g., Morbidelli et al. 2005; Fraser et al. 2014), also show a lack of aqueous alteration: their red coloration suggests irradiated methanol (e.g., Brown et al. 2011), which in the presence of liquid water would have been lost to aqueous chemical reactions (Neveu et al. 2017).

Beyond their higher propensity for volatile retention, which suggests intuitively that IDP-like bodies experienced a later and longer accretion than carbonaceous chondrite parent bodies at higher heliocentric distances, it is the fact that the outer shells of these bodies (first $\sim 20-40 \mathrm{~km}$ below the surface) never witnessed liquid water that may place a more precise constraint on the timing of their accretion. So far, this timing has not been pinpointed beyond the looser constraints that volatiles must have been retained (Jewitt et al. 2007) and that a decreasing protoplanetary disk surface density with heliocentric distance beyond the snow line results in slower accretion of more distant objects (Kenyon \& Luu 1998; Kenyon et al. 2008).

Here, we show with thermal evolution simulations that IDPlike bodies (P- D-, and some C-types) must have finished accreting at least 5-6 Myr after CAIs in order to have retained an outermost layer containing silicates unaltered by any deeper melting of ice. We also simulate the thermal evolution of CMlike bodies and confirm that the time of accretion of their outer layers - those observed today-is constrained to 3-4 Myr after CAIs. These modeling results, combined with previous telescopic observations and analyses of meteorites, confirm that the duration of planetesimal formation - at least for the last generation of bodies, which accounts for the majority of the observed small bodies and infall mass on Earth as ordinary chondrites, CM chondrites, and IDPs-steadily increased with heliocentric distance and that its influence on the compositional distribution of small bodies has not been strongly overprinted by any subsequent orbital migration.

This paper is structured as follows. In Section 2, we describe the thermal evolution code used for our calculations. We present results for carbonaceous chondrite and IDP-like parent bodies in Section 3, and discuss implications of these results in Section 4.

\section{Thermal Evolution Model}

We model the thermal evolution of both volatile-rich and CM-like planetesimals with radius $R \approx 100 \mathrm{~km}$, with accreted ${ }^{26} \mathrm{Al}$ abundances ranging from negligible (accretion many $e$-folding times past its $0.7 \mathrm{Myr}$ half-life) to those corresponding to an accretion $2 \mathrm{Myr}$ after the formation of CAIs. We model planetesimals as objects with a porosity-free density of $1955 \mathrm{~kg} \mathrm{~m}^{-3}$, corresponding to a $70 \%$ rock-30\% ice composition by mass, and a starting bulk porosity of 0.4 (starting overall density of $1173 \mathrm{~kg} \mathrm{~m}^{-3}$ ). For comparison, the bulk densities of CM chondrites, between 1800 and $2500 \mathrm{~kg} \mathrm{~m}^{-3}$ (Macke et al. 2011), suggest a similar value for those of their parent bodies, or perhaps slightly lower if ejected CM material experienced partial volatile loss. This is consistent with telescopic observations of the spectrally CM-like asteroids (Ch- and Cgh-types) for which bulk densities have been measured sufficiently precisely as to constrain their bulk composition, with values in the range of $1600-2300 \mathrm{~kg} \mathrm{~m}^{-3}$ (Carry 2012).

The evolution of internal temperatures and structure is computed numerically using a one-dimensional code (Desch et al. 2009). The planetesimals are assumed to be made of rock (idealizing a mixture of refractory materials such as silicates, metals, and carbonaceous material) and ice (water with 2 mass\% ammonia) in proportions set by the bulk density. They are assumed to accrete homogeneously, at the equilibrium temperature corresponding to their distance from the (presentday luminosity) Sun, with accretional heating assumed negligible.

Simulations start once the objects are fully formed, neglecting the progressive accretion of material over time. Material accreted after $\mathrm{CAI}+2 \mathrm{Myr}$ is never hot enough in our simulations for rock-metal differentiation, which is neglected. Any material accreted earlier would comprise only a small fraction of $100 \mathrm{~km}$-class bodies (about $1 \%$ for material at radii $<20-25 \mathrm{~km}$ and $10 \%$ for material $<45 \mathrm{~km}$ ). These deviations are smaller than those expected from varying thermo-mechanical properties of rock and ice within reasonable ranges of uncertainty.

Mass is distributed assuming spherical symmetry on a fixedvolume one-dimensional grid, with a specified number of zones (here, 200) evenly distributed in radius. The internal energy in each grid zone is computed from the initial temperature, here assumed homogeneous, using equations of state for rock and ice. A thermal structure is determined by balancing conductive heat transfer with primarily radiogenic heating by ${ }^{26} \mathrm{Al}$ and the long-lived radionuclides ${ }^{40} \mathrm{~K},{ }^{232} \mathrm{Th},{ }^{235} \mathrm{U}$, and ${ }^{238} \mathrm{U}$, using a finite-difference method and a $50 \mathrm{yr}$ time step, for 5 Gyr. Initial radionuclide abundances are provided in Table 1 . The effect of short-lived radionuclides other than ${ }^{26} \mathrm{Al}$ (e.g., ${ }^{60} \mathrm{Fe}$ and ${ }^{53} \mathrm{Mn}$ ) is neglected. Combined heating by these nuclides could be as high as that due solely to ${ }^{26} \mathrm{Al}$ depending on the bulk rock composition (e.g., Travis et al. 2012), but this could be offset by our assumed relatively high $\mathrm{Al}$ abundance of $1.5 \%$ by mass of rock (after Lodders 2003), at the upper end of the range for chondrites (0.8\%-1.75\%; Wasson \& Kallemeyn 1988). A factor of two variation in abundance is equivalent to shifting the time of accretion by the radionuclide half-life, $\sim 1$ Myr. Thermal conductivities depend on composition, temperature, and porosity.

Porosity can compact at rates determined from material viscosities (Neveu \& Rhoden 2017). Volume changes due to ice melting or freezing are neglected. Convection can occur in 
Table 1

Short- and Long-lived Radionuclide Parameters Adopted in the Thermal Evolution Model

\begin{tabular}{|c|c|c|c|c|c|}
\hline \multirow{2}{*}{ Radionuclide } & \multirow{2}{*}{$\begin{array}{l}\text { Half-life } \\
\text { (Gyr) }\end{array}$} & \multirow{2}{*}{$\begin{array}{c}\text { Initial Abundance } \\
\text { ppb by Mass of Rock }\end{array}$} & \multirow{2}{*}{$\begin{array}{l}\text { Energy Per Decay } \\
\mathrm{J}(\mathrm{kg} \text { nuclide })^{-1}\end{array}$} & \multicolumn{2}{|c|}{ Initial Specific Heat Production Rate } \\
\hline & & & & $\mathrm{W}$ (kg nuclides) $^{-1}$ & $\mathrm{~W}(\mathrm{~kg} \text { rock })^{-1}$ \\
\hline${ }^{26} \mathrm{Al}^{\mathrm{a}, \mathrm{b}}$ & 0.000716 & 724 & $1.15 \times 10^{13}$ & 0.352 & $2.55 \times 10^{-7}$ \\
\hline${ }^{40} \mathrm{~K}^{\mathrm{b}, \mathrm{c}}$ & 1.265 & 1389 & $1.46 \times 10^{12}$ & $2.53 \times 10^{-5}$ & $3.52 \times 10^{-11}$ \\
\hline${ }^{232} \mathrm{Th}^{\mathrm{b}, \mathrm{c}}$ & 14.0 & 67.59 & $1.61 \times 10^{13}$ & $2.52 \times 10^{-5}$ & $1.71 \times 10^{-12}$ \\
\hline${ }^{235} \mathrm{U}^{\mathrm{b}, \mathrm{c}}$ & 0.704 & 9.21 & $1.74 \times 10^{13}$ & $5.44 \times 10^{-4}$ & $5.01 \times 10^{-12}$ \\
\hline${ }^{238} \mathrm{U}^{\mathrm{b}, \mathrm{c}}$ & 4.47 & 29.49 & $1.85 \times 10^{13}$ & $9.11 \times 10^{-5}$ & $2.69 \times 10^{-12}$ \\
\hline
\end{tabular}

Notes.

a Castillo-Rogez et al. (2007).

${ }^{\mathrm{b}}$ Lodders (2003).

${ }^{c}$ Desch et al. (2009).

the ice shell and/or fractured core (hydrothermal circulation) if the Rayleigh number appropriate for convection between two plates or for porous media, respectively, exceeds a critical value (Neveu et al. 2015). In convective grid zones, an effective thermal conductivity is computed through multiplication by the Nusselt number (ratio of convective to conductive heat fluxes, a function of the Rayleigh number; Desch et al. 2009). In liquid grid zones, an effective thermal conductivity is set to $400 \mathrm{~W} \mathrm{~m}{ }^{-1} \mathrm{~K}^{-1}$, high enough to yield a nearly isothermal liquid layer, yet sufficiently low to satisfy the Courant criterion.

Instantaneous differentiation happens first in the innermost (warmest) grid zones if the eutectic temperature of the $\mathrm{H}_{2} \mathrm{O}-\mathrm{NH}_{3}$ system $(176 \mathrm{~K})$ is exceeded, assuming that sufficiently large rock grains settle via Stokes flow on timescales smaller than one time step. It then proceeds outward if the interior keeps warming. This yields a gravitationally unstable structure: the topmost undifferentiated layers are denser than underlying layers, which are poorer in rock. Therefore, if the body is differentiated out to more than $R / 2$, differentiation is assumed to proceed by gravitational (Rayleigh-Taylor) instabilities: layers overturn if their viscosity is below a threshold that corresponds to $T \approx 140 \mathrm{~K}$ (Rubin et al. 2014). Differentiation is assumed to be incomplete, with $25 \%$ (volatile-rich bodies) or $\geqslant 90 \%$ (CMlike asteroids; Bland \& Travis 2017) of rock assumed to be sufficiently fine-grained to remain suspended in melted and refrozen grid zones (Neveu \& Desch 2015). The rheology of the rock-ice mixture (and, therefore, its ability to overturn) is assumed to be controlled by pure water ice as long as the volume fraction of ice in rock does not exceed the close packing fraction of rock grains $(60-65 \mathrm{vol} \%$ rock). This is the case for all simulations here.

Surface temperatures are assumed to be $100 \mathrm{~K}$ or $60 \mathrm{~K}$, i.e., the equilibrium temperatures for objects with an albedo of 0.05 at about 8 and 20 au from the present-day Sun (or 6.5 and 18 au from an early Sun with $75 \%$ the present-day luminosity). These correspond to the presumed formation regions of the volatile-rich main-belt asteroids and Trojans/ Kuiper Belt Objects, respectively (Johansen et al. 2015). For CM-like bodies, the surface temperature is set to $140 \mathrm{~K}$, consistently with a presumed accretion at 3.5 to 4 au (Desch et al. 2018). We also perform runs with a $100 \mathrm{~K}$ surface to assess the effect of heliocentric distance on their thermal evolution.

\section{Results}

\subsection{IDP-like C/P/D-Type Asteroids and Jupiter Trojans}

Example results of thermal evolution simulations are shown in Figure 1 for objects with surface temperatures of $100 \mathrm{~K}$ (IDP-like C/P/D-type asteroids; Figure 1(a)) and $60 \mathrm{~K}$ (Trojans/Centaurs/KBOs; Figure 1(b)). In a typical simulation, the object undergoes a first phase of intense heating within the first $10 \mathrm{Myr}$ after its accretion (Figures 1(c), (d)), due to the decay of ${ }^{26} \mathrm{Al}$. Subsequently, it cools to relatively homogeneous temperatures of $<100-200 \mathrm{~K}$ by the present day, as it is generally too small for heat from long-lived radionuclide decay to accumulate and result in a second temperature peak. These results are consistent with thermal evolution models of outer solar system bodies akin to Saturn's captured moon Phoebe (radius $\approx 100 \mathrm{~km}$; density $1670 \mathrm{~kg} \mathrm{~m}^{-3}$; Castillo-Rogez et al. 2019).

The extent of differentiation thus depends on the amplitude of the short-lived radiogenic heat peak, such that the persistence of an anhydrous surface hinges on late accretion. As shown in Figures 1(b), 1(d), and 2, on $R \approx 100 \mathrm{~km}$ objects at $20 \mathrm{au}$, a formation $\geqslant 5 \mathrm{Myr}$ after CAIs allows the persistence of a $\gtrsim 30 \mathrm{~km}$ undifferentiated crust to the present day. Thinner crusts (Figures 1(a), (d)) are expected to undergo significant mixing or overturn due to impact bombardment.

Computed crustal thicknesses as a function of size and time of accretion are shown in Figure 2 . Only objects with $R \approx 100$ $\mathrm{km}$ formed after 5-6 Myr can retain an anhydrous surface. This suggests that IDP-like small bodies in this size range and bearing evidence of dry silicates finished forming after $5 \mathrm{Myr}$.

We do not expect any other potential later-stage heating process to affect the persistence of surface anhydrous material. Such processes include large-scale planetary migration induced by gravitational perturbations, in which objects may have moved closer in ( $5 \mathrm{au}$ for Trojans) in the first gigayear after formation (Morbidelli et al. 2005). Indeed, inside volatile-rich objects, the mud layer surrounding the rocky core remains nearly as cold as the surface past $0.5 \mathrm{Gyr}$, irrespectively of heliocentric distance (Figures 1(a), (b)). Likewise, warming by impacts is not expected to exceed a few kelvin globally given likely projectile kinetic energies of $10^{22}-10^{23} \mathrm{~J}$ (Wilhelms \& Squyres 1984; Marinova et al. 2011).

Instead of primordial undifferentiated crust, the anhydrous material might be fine rock grains suspended in "frozen mud" overturned by Rayleigh-Taylor instabilities below the melting point of $\mathrm{H}_{2} \mathrm{O}\left(-\mathrm{NH}_{3}\right)$ ice. However, melting must occur at depth in order to initiate the differentiation of the central regions 

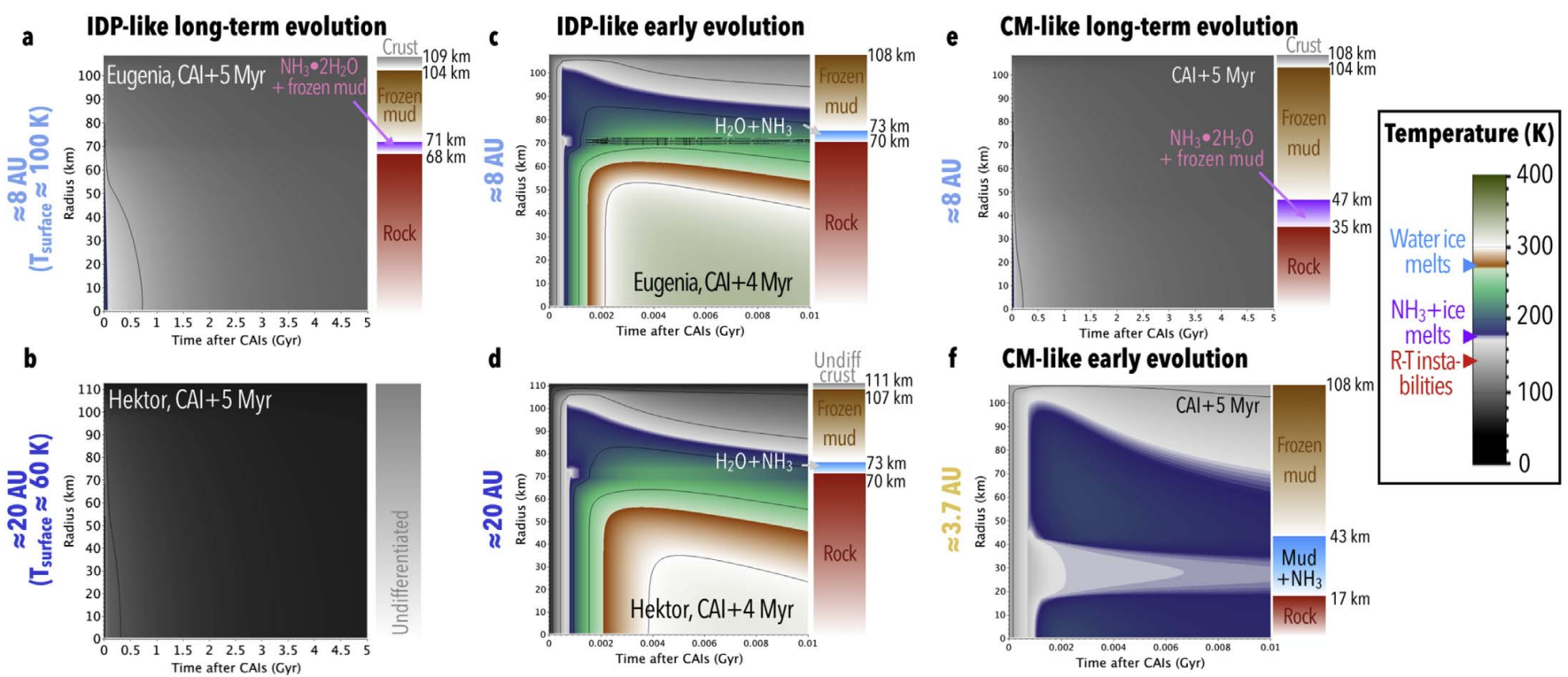

Figure 1. Panels (a)-(b): thermal evolution of $R=100 \mathrm{~km}$-class volatile-rich objects in limit cases of ice-rock differentiation. Indicative heliocentric distances are shown to the left of each plot. Internal structures at the present day $(4.56 \mathrm{Gyr})$ are shown as bars to the right of each temperature plot. The time of accretion in megayears is indicated in the top left corner of each panel, along with the indicative name of a solar system body of similar size and possible place of accretion. Panels (c)-(d): first $10 \mathrm{Myr}$ of the thermal evolution of volatile-rich objects assuming accretion $4 \mathrm{Myr}$ after CAIs. Internal structures after $10 \mathrm{Myr}$ are shown as bars to the right of each temperature plot. Panels (e)-(f): long-term and early thermal evolution of CM-like objects. Even for late-accreting objects at 8 au, internal structures (bars to the right) at $4.56 \mathrm{Gyr}$ suggest that an anhydrous crust thick enough to withstand impacts cannot persist.

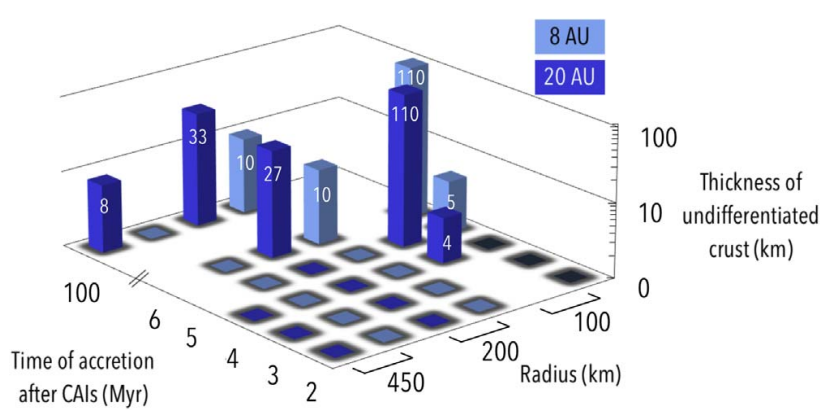

Figure 2. Thickness of the undifferentiated crust of IDP-like bodies as a function of radius and time of formation. Numbers indicate the thickness in kilometers of the undifferentiated outer layer (simulation result). Crusts thinner than $20-30 \mathrm{~km}$ are likely overturned by impacts.

necessary to yield a gravitationally unstable configuration. Indeed, deep liquid water persists for $\geqslant 10$ Myr (Figures 1(c), (d)), and convectively overturned material would have likely been sourced from (or at least come in contact with) such deeper melted material and undergone some degree of hydration. Therefore, we deem it unlikely that endogenous surface silicates can remain dry unless the surface material has remained undifferentiated.

\subsection{CM-like Asteroids and CM Parent Bodies}

Simulation results depicting the computed evolution of CMlike objects are shown in Figures 1(e), (f). These objects invariably fully overturn (crust at most a few kilometers thick), owing to the more homogeneous distribution of temperatures inherent to their assumed "mudball" interior, comprised of a small rocky core overlain by a thick mud layer (Bland \& Travis 2017), which is convective early on when differentiation takes place. At $3.7 \mathrm{au}$, the surface is at $140 \mathrm{~K}$ and the interior necessarily warmer, so Rayleigh-Taylor instabilities can overturn any gravitationally unstable configuration generated by deep melting. This occurs even by long-lived radionuclide heating alone $\left(\right.$ no $\left.{ }^{26} \mathrm{Al}\right)$; only objects with a $100 \mathrm{~K}$ surface and without ${ }^{26} \mathrm{Al}$ can maintain a thick crust over the age of the solar system.

For aqueous alteration to take place, CM-like objects must accrete early. Unless these objects accrete past 6 Myr after CAIs, the warmest temperatures are experienced during the first $10 \mathrm{Myr}$ of their evolution, owing to the decay of ${ }^{26} \mathrm{Al}$. For $R \approx 100 \mathrm{~km}$ bodies, $\mathrm{H}_{2} \mathrm{O}-\mathrm{NH}_{3}$ ice melts only for accretion $<5 \mathrm{Myr}$ after CAIs, and reaches several dozen ${ }^{\circ} \mathrm{C}$ (the alteration temperatures inferred from CM chondrite analyses; Guo \& Eiler 2007; Cody et al. 2008) for accretion around $3 \mathrm{Myr}$ after CAIs. To melt pure $\mathrm{H}_{2} \mathrm{O}$ ice, accretion must take place before $4 \mathrm{Myr}$ after CAIs. Thus, the presence of aqueously altered material at the surface of CM-like asteroids suggests that these objects completed accretion no later than 3-4 Myr after CAIs. This is consistent with constraints from the meteorite record (Sugiura \& Fujiya 2014, their Figure 2).

Note that in the first $0.01 \mathrm{Gyr}$ (Figure 1(f)), the liquid layer ("Mud+ $\mathrm{NH}_{3}$ ") is slightly colder than the overlying "frozen mud" layer composed of rock and $\mathrm{H}_{2} \mathrm{O}$ ice (it is assumed that $\mathrm{NH}_{3}$ remains in the liquid upon partial freezing until the eutectic composition is reached). At this early time, internal energy is still relatively homogeneous among all grid zones. Because the liquid layer is near freezing, part of its internal energy is used as latent heat in keeping $\mathrm{H}_{2} \mathrm{O}$ and $\mathrm{NH}_{3}$ melted, whereas in the solid layers above and below, all of the internal energy is used to elevate their temperature above $0 \mathrm{~K}$. During this brief time, the heat flux across the base of the frozen mud layer, driven by temperature differences, is positive going inward. In IDP-like body simulations, this stage also occurs but temperatures revert even more quickly (within $\approx 1 \mathrm{Myr}$ ) to a monotonous gradient (colder going outward) because of the more concentrated radiogenic heat production in the larger core. 


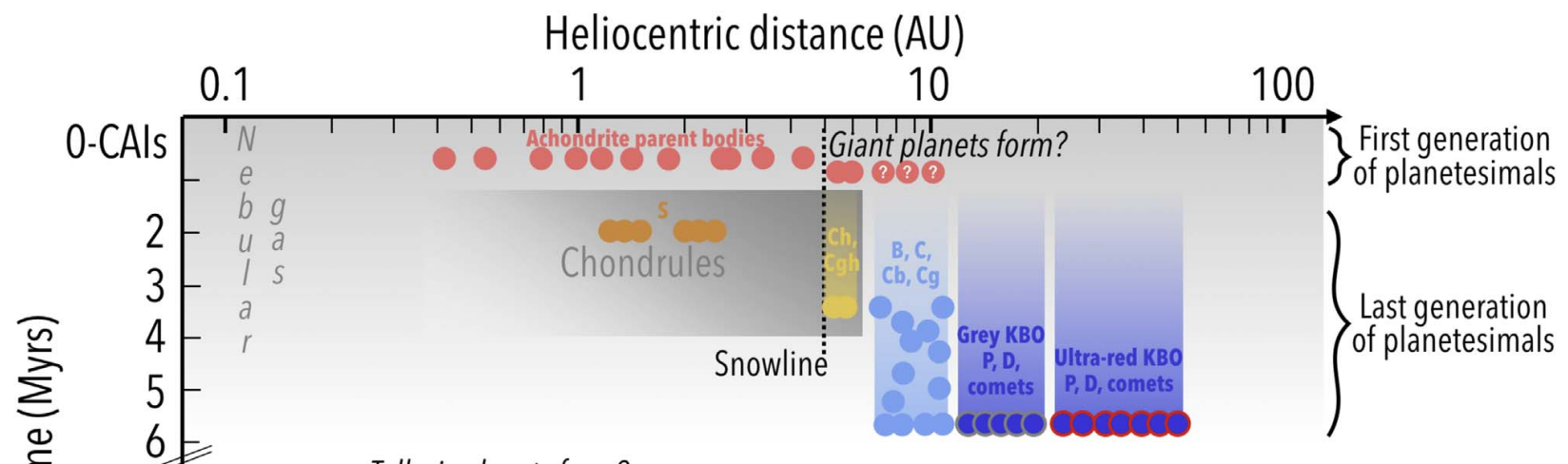

Telluric planets form?

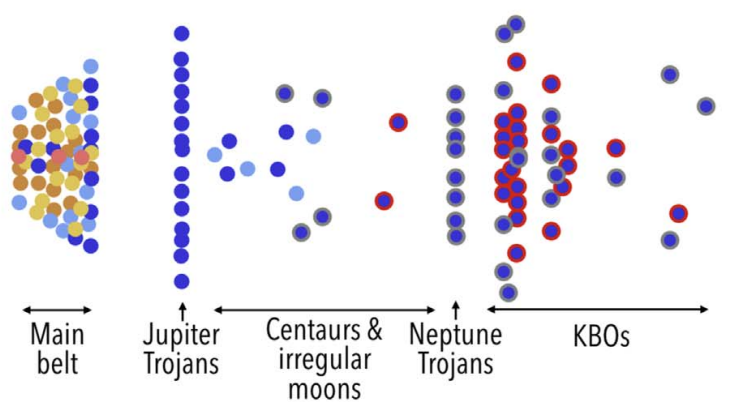

Figure 3. Postulated sequence of events tracing the time, place, and duration of formation of small bodies (top) to present-day observed characteristics (bottom; vertical spread reproducing roughly the distribution of orbital inclinations). The accretion duration is shown as gradient boxes ending at the fully formed bodies. Meteorite analyses indicate that achondrite parent bodies began accreting before 2 Myr even beyond the snow line. Their formation is depicted as occurring slightly earlier in the inner solar system compared to beyond $5 \mathrm{au}$; this is speculative and solely based on mirroring the trend inferred for the second generation of bodies. Our simulations suggest that volatile-rich bodies accreted their outer layers after 5-6 Myr, but the onset of their accretion is unconstrained if these are a separate generation of bodies.

\section{Discussion}

In Figure 3, we use the results of our simulations along with those obtained for the formation time of inner solar system S-type asteroids (Monnereau et al. 2013) to depict our current understanding of the time and duration of formation of the different compositional classes of $D \approx 200 \mathrm{~km}$ bodies as a function of heliocentric distance. The likely-yet not firmly established-formation locations for the different classes of small bodies were retrieved from Vernazza \& Beck (2017). It appears that the timescale of completion of formation of the last generation of small $D \leqslant 200 \mathrm{~km}$ bodies (in megayears) is positively correlated with their formation location (in astronomical units). Accretion ended earlier inward of the snow line $(\approx 2 \mathrm{Myr}$ for ordinary chondrite parent bodies; Henke et al. 2012; Monnereau et al. 2013; Blackburn et al. 2017) than just beyond Jupiter (3-4 Myr, CM-like bodies), which itself stopped earlier than for objects farther out $(>5 \mathrm{Myr}$ for IDPlike bodies at $\approx 8 \mathrm{au}$ and $>6 \mathrm{Myr}$ at $\approx 20 \mathrm{au}$ ).

Meteoritic evidence suggests the existence of at least one previous generation of planetesimals, which would also have formed later at higher heliocentric distances (Kruijer et al. 2017). In the inner solar system, this first generation comprises the parent bodies of differentiated achondrites (Russell et al. 2006). Beyond the snow line, it includes achondritic meteoritic material (groups IIC, IID, IIF, IIIF, and IVB of iron meteorites) isotopically related to carbonaceous chondrites and IDPs. Inferred accretion times for these objects suggest that the onset of planetesimal formation also seems to have occurred later beyond the snow line $(\approx 1 \mathrm{Myr}$; Kruijer et al. 2017) than inward of it ( $\approx 0.2 \mathrm{Myr})$. Thus, the accretion of $D \approx 200 \mathrm{~km}$ bodies seems to have both started and ended later at greater heliocentric distances, even though the periods of accretion inside (0.2-2 Myr) and beyond the snow line (1-6+ Myr) overlapped.

Although the meteorite record has also been interpreted in terms of continuous accretion over these time ranges, this likely concerns only a small fraction of the mass of the small body populations as inferred from telescopic observations (types other than $\mathrm{S} / \mathrm{C} / \mathrm{P} / \mathrm{D}$ ) and the astromaterial record (primitive achondrites). In this interpretation, some bodies accreted early enough to have experienced at least partial dehydration (Weiss \& Elkins-Tanton 2013). The latter case is not supported by current measurements of the spectral properties and densities of $D>150 \mathrm{~km}$ CM-like bodies (Carry 2012; Vernazza et al. 2016; Vernazza \& Beck 2017; Carry et al. 2019), whose low values are more compatible with a mostly or fully homogenous interior.

Also arguing against accretion sustained at a continuous pace over this period is the (albeit possibly biased) scant representation of observed bodies (e.g., A-type and K-type bodies) related to meteorites of intermediate thermal alteration stages such as CV and CK chondrites (DeMeo \& Carry 2013). Likewise, primitive achondrites of intermediate age are underrepresented in the meteorite collection, even compared to differentiated achondrites from the earliest planetesimals (Meteoritical Bulletin Database 2018). Therefore, we do not exclude that the objects simulated here could have accreted over several megayears (Figure 3; colored gradient boxes starting at $\approx 2 \mathrm{Myr}$ ), provided that the bulk of accretion took place sufficiently late as to avoid any significant thermal alteration and rock-metal differentiation. 
The delay or extension of the accretional period beyond the snow line may be rooted in a much lower initial surface density of the protoplanetary disk there than inward of the snow line, at least for a few megayears until the disk density at 5-10 au increased due to outward viscous spreading (e.g., Desch et al. 2018). Assuming that the small body accretion timescale was proportional to the surface density in the accretion disk of the Sun and in proportion to the Keplerian orbit period (i.e., accretion duration proportional approximately to the cube of the heliocentric distance; Jewitt et al. 2007), the formation of IDP-like bodies may have taken much longer (tens of megayears) than the lower bound of 5-6 Myr implied by our results. The strong time dependence of accretion on heliocentric distance breaks down at several tens of astronomical units, where the accretion timescale is longer than that of orbital velocity dispersion excitation to the point where collisions become destructive (Kenyon \& Luu 1998; Jewitt et al. 2007; Schlichting \& Sari 2011), placing an upper bound of $<100$ Myr to form 100 kilometer-class Kuiper Belt objects.

The accretion timescales inferred from this study confirm that there has not been a universal formation process for solar system bodies. Indeed, Jupiter must have reached a significant size by 3 Myr or even earlier ( $\leqslant 1$ Myr following Kruijer et al. 2017 ) in order to retain its massive gaseous envelope and create a gap in the disk that would naturally explain the isotopic dichotomy between enstatite/ordinary chondrites and carbonaceous chondrites (e.g., Warren 2011; Budde et al. 2016), at a time when our results suggest IDP-like P- and D-type bodies were not yet formed. The same applies to the remaining giant planets that must have accreted before dissipation of the gas. This illustrates the fact that specific locations in the disk such as the snow line favored the rapid formation of large, massive bodies (giant planet cores) whereas other formation regions only allowed the formation of small $(D \sim 200 \mathrm{~km}$ ) bodies (e.g., formation region of IDP-like bodies) at a slow pace (a few megayears to grow from dust particles to $\sim 200 \mathrm{~km}$ ). Such a slow pace of accretion for distant small bodies is consistent with the late accretion of comet 67P (Mousis et al. 2017) and the presence of many contact binaries among KBOs (Thirouin \& Sheppard 2018), including the KBO Ultima Thule recently flown by the New Horizons mission.

The completion of IDP-like P- and D-type asteroid accretion past 5-6 Myr also makes it less likely that the pebble accretion (Levison et al. 2015a, 2015b) and Grand Tack scenarios (Walsh et al. 2011) can describe the accretion of the bulk of their mass and their early migration (if any), respectively. Indeed, these models require nebular gas, which would likely have disappeared by 5 Myr (Walsh et al. 2012), although a few extrasolar disks have been observed to persist until $10 \mathrm{Myr}$ (Russell et al. 2006). If these scenarios apply, our results strongly constrain the time window in which they should have operated (and in which IDP-like bodies finished accreting) to CAI +5-6 Myr, assuming nebular gas persisted long enough.

The authors contributed equally to this article. We thank an anonymous reviewer for comments that helped improve this article. M.N. thanks S. Desch for providing access to the computers on which simulations were run.

Software: IcyDwarf (https://github.com/MarcNeveu/Icy Dwarf).

\section{ORCID iDs}

Marc Neveu (ib https://orcid.org/0000-0002-6220-2869

\section{References}

Bennett, M. E., \& McSween, H. Y. 1996, M\&PS, 31, 783

Bischoff, A. 1998, M\&PS, 33, 1113

Blackburn, T., Alexander, C. M. O., Carlson, R., \& Elkins-Tanton, L. T. 2017, GeCoA, 200, 201

Bland, P. A., \& Travis, B. J. 2017, SciA, 3, e1602514

Bradley, J. P. 2003, in Treatise on Geochemistry, Vol. 1 ed. H. D. Holland \& K. K. Turekian (Amsterdam: Elsevier), 1

Brown, M. E., Schaller, E. L., \& Fraser, W. C. 2011, ApJL, 739, L60

Budde, G., Burkhardt, C., Brennecka, G. A., et al. 2016, E\&PSL, 454, 293

Burbine, T. H., McCoy, T. J., Meibom, A., Gladman, B., \& Keil, K. 2002, in Asteroids III, ed. W. Bottke et al. (Tucson, Arizona: Univ. Arizona Press), 653

Campins, H., Hargrove, K., Pinilla-Alonso, N., et al. 2010, Natur, 464, 1320 Carry, B. 2012, P\&SS, 73, 98

Carry, B., Vachier, F., Berthier, J., et al. 2019, A\&A, 623, A132

Castillo-Rogez, J., Matson, D., Sotin, C., et al. 2007, Icar, 190, 179

Castillo-Rogez, J., Vernazza, P., \& Walsh, K. 2019, MNRAS, in press

Cloutis, E. A., Binzel, R. P., \& Gaffey, M. J. 2014, Elements, 10, 25

Cody, G., Yabuta, H., Kilcoyne, A., et al. 2008, E\&PSL, 272, 446

Cohen, B. A., \& Coker, R. F. 2000, Icar, 145, 369

DeMeo, F. E., \& Carry, B. 2013, Icar, 226, 723

Desch, S. J., Cook, J. C., Doggett, T., \& Porter, S. B. 2009, Icar, 202, 694

Desch, S. J., Kalyaan, A., \& Alexander, C. M. O. 2018, ApJS, 238, 11

Emery, J. P., Cruikshank, D. P., \& Van Cleve, J. 2006, Icar, 182, 496

Emery, J. P., Marzari, F., Morbidelli, A., French, L. M., \& Grav, T. 2015, in Asteroids IV, ed. P. Michel, F. E. DeMeo, \& W. F. Bottke (Tucson, AZ: Univ. Arizona Press), 203

Fraser, W. C., Brown, M. E., Morbidelli, A., Parker, A., \& Batygin, K. 2014 ApJ, 782, 100

Greenwood, R. C., Burbine, T. H., Miller, M. F., \& Franchi, I. A. 2017, ChEG, 77, 1

Grimm, R. E., \& McSween, H. Y. 1989, Icar, 82, 244

Guo, W., \& Eiler, J. M. 2007, GeCoA, 71, 5565

Hargrove, K. D., Kelley, M. S., Campins, H., Licandro, J., \& Emery, J. 2012, Icar, 221, 453

Harvey, R., \& Cassidy, W. 1989, Metic, 24, 9

Henke, S., Gail, H.-P., Trieloff, M., Schwarz, W. H., \& Kleine, T. 2012, A\&A, 545, A135

Howard, K., Benedix, G., Bland, P., \& Cressey, G. 2009, GeCoA, 73, 4576

Howard, K., Benedix, G., Bland, P., \& Cressey, G. 2011, GeCoA, 75, 2735

Jewitt, D., Chizmadia, L., Grimm, R., \& Prialnik, D. 2007, in Protostars and Planets V, ed. B. Reipurth, D. Jewitt, \& K. Keil (Tucson, AZ: Univ. Arizona Press), 863

Johansen, A., Mac Low, M.-M., Lacerda, P., \& Bizzarro, M. 2015, SciA, 1, e1500109

Johnson, T. V., \& Fanale, F. P. 1973, JGR, 78, 8507

Kenyon, S. J., Bromley, B. C., OÕBrien, D. P., \& Davis, D. R. 2008, The Solar System Beyond Neptune (Tucson, Arizona: Univ. Arizona Press), 293

Kenyon, S. J., \& Luu, J. X. 1998, AJ, 115, 2136

Kruijer, T. S., Burkhardt, C., Budde, G., \& Kleine, T. 2017, PNAS, 114, 6712 Lauretta, D. S., Bartels, A. E., Barucci, M. A., et al. 2015, M\&PS, 50, 834

Levison, H. F., Kretke, K. A., \& Duncan, M. J. 2015a, Natur, 524, 322

Levison, H. F., Kretke, K. A., Walsh, K. J., \& Bottke, W. F. 2015b, PNAS, 112,14180

Licandro, J., Campins, H., Kelley, M., et al. 2011, A\&A, 525, A34

Liou, J.-C., Zook, H. A., \& Dermott, S. F. 1996, Icar, 124, 429

Lodders, K. 2003, ApJ, 591, 1220

Lodders, K., \& Osborne, R. 1999, Composition and Origin of Cometary Materials (Berlin: Springer), 289

Love, S. G., \& Brownlee, D. E. 1993, Sci, 262, 550

Macke, R. J., Consolmagno, G. J., \& Britt, D. T. 2011, M\&PS, 16, 1842

Marchis, F., Durech, J., Castillo-Rogez, J., et al. 2014, ApJL, 783, L37

Marinova, M. M., Aharonson, O., \& Asphaug, E. 2011, Icar, 211, 960

Meteoritical Bulletin Database 2018, https://www.lpi.usra.edu/meteor retrieved Dec. 20

Monnereau, M., Toplis, M. J., Baratoux, D., \& Guignard, J. 2013, GeCoA, 119,302

Morbidelli, A., Levison, H. F., Tsiganis, K., \& Gomes, R. 2005, Natur, 435, 462

Mousis, O., Drouard, A., Vernazza, P., et al. 2017, ApJL, 839, L4

Nakamura, T., Noguchi, T., Tanaka, M., et al. 2011, Sci, 333, 1113 
Neveu, M., \& Desch, S. J. 2015, GeoRL, 42, 10, 197

Neveu, M., Desch, S. J., \& Castillo-Rogez, J. C. 2015, JGRE, 120, 123

Neveu, M., Desch, S. J., \& Castillo-Rogez, J. C. 2017, GeCoA, 212, 324

Neveu, M., \& Rhoden, A. R. 2017, Icar, 296, 183

Pajuelo, M., Carry, B., Vachier, F., et al. 2018, Icar, 309, 134

Perna, D., Barucci, M. A., Ishiguro, M., et al. 2017, A\&A, 599, L1

Pieters, C. M., \& McFadden, L. A. 1994, AREPS, 22, 457

Rivkin, A. S., \& Emery, J. P. 2010, Natur, 464, 1322

Rosenberg, N. D., Browning, L., \& Bourcier, W. L. 2001, M\&PS, 36, 239

Rubin, M. E., Desch, S. J., \& Neveu, M. 2014, Icar, 236, 122

Russell, S. S., Hartmann, L., Cuzzi, J., et al. 2006, in Meteorites and the Early

Solar System II, ed. D. S. Lauretta \& H. Y. McSween Jr. (Tucson, AZ:

Univ. Arizona Press), 233

Sandford, S. A., \& Bradley, J. P. 1989, Icar, 82, 146

Schlichting, H. E., \& Sari, R. 2011, ApJ, 728, 68

Sugiura, N., \& Fujiya, W. 2014, M\&PS, 49, 772

Takir, D., \& Emery, J. P. 2012, Icar, 219, 641

Thirouin, A., \& Sheppard, S. S. 2018, AJ, 155, 248

Tomeoka, K., \& Buseck, P. R. 1985, GeCoA, 49, 2149

Travis, B., Palguta, J., \& Schubert, G. 2012, Icar, 218, 1006
Urey, H. C. 1955, PNAS, 41, 127

Usui, F., Hasegawa, S., Ootsubo, T., \& Onaka, T. 2019, PASJ, 71,

Vernazza, P., \& Beck, P. 2017, in Planetesimals: Early Differentiation and Consequences for Planets, ed. L. T. Elkins-Tanton \& B. P. Weiss (Cambridge: Cambridge Univ. Press), 269

Vernazza, P., Castillo-Rogez, J., Beck, P., et al. 2017, AJ, 153, 72

Vernazza, P., Delbo', M., King, P. L., et al. 2012, Icar, 221, 1162

Vernazza, P., Marsset, M., Beck, P., et al. 2015, ApJ, 806, 204

Vernazza, P., Marsset, M., Beck, P., et al. 2016, AJ, 152, 54

Vernazza, P., Zanda, B., Binzel, R. P., et al. 2014, ApJ, 791, 120

Walsh, K. J., Morbidelli, A., Raymond, S. N., O'Brien, D. P., \& Mandell, A. M. 2011, Natur, 475, 206

Walsh, K. J., Morbidelli, A., Raymond, S. N., O'Brien, D. P., \& Mandell, A. M. 2012, M\&PS, 47, 1941

Warren, P. H. 2011, E\&PSL, 311, 93

Wasson, J. T., \& Kallemeyn, G. W. 1988, RSPTA, 325, 535

Weiss, B. P., \& Elkins-Tanton, L. T. 2013, AREPS, 41, 529

Wilhelms, D. E., \& Squyres, S. W. 1984, Natur, 309, 138

Young, E. D., Zhang, K. K., \& Schubert, G. 2003, E\&PSL, 213, 249

Zolensky, M., Barrett, R., \& Browning, L. 1993, GeCoA, 57, 3123 